\title{
DISTANCE ESTIMATES FOR VON NEUMANN ALGEBRAS
}

\author{
SHLOMO ROSENOER
}

ABSTRACT. It is shown that for certain von Neumann algebras $\mathcal{Q}$, there is a constant $C$ such that

$$
\operatorname{dist}(T, \mathbb{Q}) \leqslant C \sup _{P \in \text { lat } \mathbb{Q}}\left\|P^{\perp} T P\right\| \quad \text { for all } T \text { in } \mathscr{B}(\mathcal{C}) \text {. }
$$

1. Introduction. Throughout this paper, $\mathcal{H}$ denotes a separable Hilbert space and $\mathscr{B}(\mathcal{H C})$ is the algebra of all bounded linear operators on $\mathcal{H}$. For any subalgebra $\mathcal{Q}$ of $\mathscr{B}(\mathcal{H})$, let lat $\mathbb{Q}$ denote the lattice of orthogonal projections $P$ invariant for $\mathcal{Q}$. That is, $P^{\perp} A P=0$ for all $A$ in $Q$, where $P^{\perp}=I-P$. $Q$ is said to be reflexive if every operator $B$ satisfying $P^{\perp} B P=0$ for all $P$ in lat $Q$ belongs to $\mathcal{Q}$.

Let $Q$ be a reflexive algebra and $T$ an arbitrary operator in $\mathscr{B}(\mathscr{H})$. It is easy to see that

$$
\operatorname{dist}(T, \mathbb{Q}) \geqslant \sup _{P \in \text { lat } \mathbb{Q}}\left\|P^{\perp} T P\right\| .
$$

Arveson [1] proved that if $Q$ is a nest algebra, then equality actually occurs in (1). Davidson [3] has referred to Choi's example which shows that equality fails to hold even if $\mathcal{H}$ is finite dimensional and $\mathcal{Q}$ is a m.a.s.a. He asked: if $\mathcal{Q}$ is reflexive and lat $\mathscr{Q}$ is commutative, then is there a constant $C$ such that

$$
\operatorname{dist}(T, \mathbb{Q}) \leqslant C \sup _{P \in \operatorname{lat} \mathbb{Q}}\left\|P^{\perp} T P\right\|
$$

for all $T$ in $\mathscr{B}(\mathcal{H})$ ?

In this paper, we shall prove that (2) holds (with $C=2$ ) if $\mathscr{Q}$ is a von Neumann algebra such that either $\mathscr{Q}$ or $\mathbb{Q}^{\prime}$ is abelian. Note that if $\mathbb{Q}^{\prime}$ is commutative, then so is lat $\mathscr{Q}$. Also if $\mathscr{Q}$ is a weakly closed unital algebra of normal operators, then (2) holds with $C=3$.

In [5], Johnson conjectured that for a von Neumann algebra $\mathscr{Q}$ there is a positive constant $K$ such that for all $T$ in $\mathscr{B}(\mathcal{H})$,

$$
\operatorname{dist}(T, \mathbb{Q}) \leqslant K\left\|\left.\Delta_{T}\right|_{\mathbb{Q}^{\prime}}\right\|
$$

where $\Delta_{T}$ is the derivation $\Delta_{T}(S)=S T-T S$. Christensen [2] proved that (3) holds for a very large class of von Neumann algebras. We will show that (2) and (3) are equivalent.

Received by the editors December 29, 1980.

1980 Mathematics Subject Classification. Primary 46L99, 47C05.

(C1982 American Mathematical Society 0002-9939/81/0000-1104/\$01.50 


\section{Distance estimates and derivations.}

THEOREM 2.1. Let $Q$ be a von Neumann algebra and let $T$ belong to $\mathscr{B}(\mathcal{H})$.

(i) If $\operatorname{dist}(T, \mathbb{Q}) \leqslant K\left\|\left.\Delta_{T}\right|_{\mathbb{Q}^{\prime}}\right\|$, then

$$
\operatorname{dist}(T, Q) \leqslant 4 K \sup _{P \in \operatorname{lat} Q}\left\|P^{\perp} T P\right\| .
$$

(ii) If $\operatorname{dist}(T, \mathbb{Q}) \leqslant C \sup _{P \in \text { lat } \mathbb{Q}}\left\|P^{\perp} T P\right\|$, then

$$
\operatorname{dist}(T, \mathscr{Q}) \leqslant \frac{C}{2}\left\|\left.\Delta_{T}\right|_{\mathbb{Q}^{\prime}}\right\| \text {. }
$$

Proof. Since lat $\mathcal{Q}$ is complemented, we have

$$
\begin{aligned}
2 \sup _{P \in \text { lat } Q}\left\|P^{\perp} T P\right\| & =2 \sup _{P \in \operatorname{lat} Q} \max \left\{\left\|P^{\perp} T P\right\|,\left\|P T P^{\perp}\right\|\right\} \\
& =2 \sup _{P \in \operatorname{lat} Q}\|P T-T P\| \\
& =\sup _{P \in \operatorname{lat} Q}\|(2 P-I) T-T(2 P-I)\| \leqslant\left\|\left.\Delta_{T}\right|_{\mathbb{Q}^{\prime}}\right\| .
\end{aligned}
$$

This proves (ii).

Now suppose $\sup _{P \in \text { lat } \mathcal{Q}}\|P T-T P\|=\delta$. Then for $P$ in lat $\mathcal{Q},\left\|\Delta_{T}(2 P-I)\right\| \leqslant$ $2 \delta$. Let $\mathfrak{N}$ be the real vector space of all Hermitian operators in $Q^{\prime}$. By the Krein-Milman theorem, the unit ball of $\mathfrak{N}$ is the weakly closed convex hull of its extreme points. But these extreme points are precisely $(2 P-I)$ for projections $P$ in $\mathcal{Q}^{\prime}$, namely lat $\mathcal{Q}$. Thus $\left\|\left.\Delta_{T}\right|_{\pi}\right\| \leqslant 2 \delta$. If $B$ in $\mathcal{Q}^{\prime}$ has $\|B\| \leqslant 1$, then write $B=A_{1}+$ $i A_{2}$ where $A_{i}$ are Hermitian and $\left\|A_{i}\right\| \leqslant 1$. Then $\left\|\Delta_{T}(B)\right\| \leqslant\left\|\Delta_{T}\left(A_{1}\right)\right\|+$ $\left\|\Delta_{T}\left(A_{2}\right)\right\| \leqslant 4 \delta$, which proves (i).

3. Abelian von Neumann algebras. In [2], it is established that if $Q^{\prime}$ is abelian,

$$
\operatorname{dist}(T, \mathbb{Q}) \leqslant\left\|\left.\Delta_{T}\right|_{\mathbb{Q}^{\prime}}\right\| \text {. }
$$

By Theorem 2.1, we conclude that (1) holds with $C=4$. In fact, we have

LEMMA 3.1. If $Q$ is a von Neumann algebra with abelian commutant, then for every $T$ in $\mathscr{B}(\mathcal{H})$,

$$
\operatorname{dist}(T, Q) \leqslant 2 \sup _{P \in \text { lat } Q}\left\|P^{\perp} T P\right\| .
$$

Proof. Let $\delta=\sup _{P \in \text { lat } Q}\left\|P^{\perp} T P\right\|$. Let $G$ be the group of unitaries generated by $\{2 P-I: P \in$ lat $Q\}$. Then for every element $U$ in $G,\|U T-T U\| \leqslant 2 \delta$, whence $\left\|T-U^{-1} T U\right\| \leqslant 2 \delta$. Since $G$ is abelian, it has an invariant mean $m$. Let $f(U)=$ $U^{-1} T U$, and define $T_{0}=m(f)$ following the method of [6]. Since $T_{0}$ is in the weakly closed convex hull of $\left\{U^{-1} T U: U \in G\right\}$, we have $\left\|T-T_{0}\right\| \leqslant 2 \delta$. But the invariance of $m$ shows that $T_{0}$ belongs to $G^{\prime}=\left(\mathbb{Q}^{\prime}\right)^{\prime}=\mathbb{Q}$. Thus $\operatorname{dist}(T, \mathbb{Q}) \leqslant 2 \delta$.

LEMMA 3.2. Let $\phi$ be a functional on $\mathscr{B}(\mathcal{H})$ continuous in the weak operator topology such that $\phi(I)=0$. Then the kernel of $\phi$ contains a m.a.s.a.

Proof. There is a finite rank operator $A$ such that $\phi(T)=\operatorname{tr}(A T)$ for all $T$ in $\mathscr{B}(\mathcal{H C})$. It suffices to find an orthonormal basis $\left\{f_{i}\right\}$ for $\mathcal{H}$ such that $\left(A f_{i}, f_{i}\right)=0$ for all $i$. For then, we take our m.a.s.a. to be all operators which are diagonal with respect to this basis. 
The numerical range of an operator $B$, namely $W(B)=\{(B x, x):\|x\|=1\}$, is always convex and nonempty. When $B$ acts on a space of finite dimension $n$, we have

$$
\frac{1}{n} \sum_{i=1}^{n}\left(B e_{i}, e_{i}\right)=\frac{1}{n} \operatorname{tr} B .
$$

So if $\operatorname{tr} B=0$, then 0 belongs to $W(B)$ and thus there is a unit vector $f$ such that $(B f, f)=0$. On the complement $\mathscr{T}$ of $\operatorname{span}\{f\}$, we have

$$
0=\operatorname{tr} B=(B f, f)+\operatorname{tr}\left(\left.B\right|_{\Re}\right)=\operatorname{tr}\left(\left.B\right|_{\Re}\right) .
$$

By induction, there is an orthonormal basis $\left\{f_{i}, 1 \leqslant i \leqslant n\right\}$ with $\left(B f_{i}, f_{i}\right)=0$.

Choose a finite dimensional subspace $\Re$ which reduces $A$ and $\left.A\right|_{\Re^{\perp}}=0$. Apply the previous paragraph to $\left.A\right|_{\Re}$ and complete the orthonormal set with an arbitrary basis for $\Re^{\perp}$.

Lemma 3.3. Let $\mathbb{Q}$ be an abelian von Neumann algebra on $\mathcal{H}$, and let $\phi$ be a weak operator continuous functional on $\mathscr{B}(\mathcal{H})$ which annihilates $\mathcal{Q}$. Then there is a m.a.s.a. Tl containing $Q$ in the kernel of $\phi$.

Proof. We shall use the direct integral decomposition of $\mathcal{Q}[\mathbf{8}, \mathrm{p} .19]$. There is a measure space $(Z, \mu)$ such that $\mathcal{K}=\int^{\oplus} \mathcal{H}(\zeta) d \mu(\zeta)$ and $\mathcal{Q}$ is the algebra of all operators $T=\int^{\oplus} T(\zeta) d \mu(\zeta)$ where $T(\zeta)$ is a scalar multiple of the identity $I_{\mathcal{Y C}(\zeta)}$. We can write $\phi$ in the form $\phi(T)=\sum_{i=1}^{n}\left(T x_{i}, y_{i}\right)$, and $x_{i}=\int^{\oplus} x_{i}(\zeta) d \mu(\zeta)$ and $y_{i}=\int^{\oplus} y_{i}(\zeta) d \mu(\zeta)$.

Define $f(\zeta)=\sum_{i=1}^{n}\left(x_{i}(\zeta), y_{i}(\zeta)\right)$. Then $f(\zeta)$ belongs to $L^{1}(Z, \mu)$. If $g(\zeta)$ is a bounded measurable function on $(Z, \mu)$, then

$$
0=\phi\left(\int^{\oplus} g(\zeta) I_{\mathcal{K C}(\zeta)} d \mu(\zeta)\right)=\int f(\zeta) g(\zeta) d \mu(\zeta)
$$

Hence $f(\zeta)=0$ a.e.

Let $\phi_{\zeta}$ be the weak operator continuous functional on $\mathscr{B}(\mathcal{H}(\zeta))$ given by $\phi_{\zeta}(S)=$ $\sum_{i=1}^{n}\left(S x_{i}(\zeta), y_{i}(\zeta)\right)$. Then $\phi_{\zeta}(I)=0$ for almost all $\zeta$. So by Lemma 3.2 , there is a m.a.s.a. $\mathfrak{N}(\zeta)$ in the kernel of $\phi_{\zeta}$. Let $\mathfrak{R}=\int^{\oplus} \mathfrak{R}(\zeta) d \mu(\zeta)$. It is easy to verify that $\mathscr{K}$ is a m.a.s.a. in $\mathscr{B}(\mathcal{K})$ and $\phi(\mathscr{N})=0$.

LEMMA 3.4. If $\mathcal{Q}$ is an abelian von Neumann algebra, and $T$ belongs to $\mathscr{B}(\mathcal{H})$, then

$$
\operatorname{dist}(T, \mathbb{Q})=\sup \operatorname{dist}(T, \mathfrak{N})
$$

where the sup is taken over all m.a.s.a.'s $\mathbb{N}$ containing $\mathbb{Q}$.

Proof. We will prove the nontrivial inequality $\operatorname{dist}(T, \mathscr{Q}) \geqslant \sup \operatorname{dist}(T, \Re)$. Let $\delta$ denote the right-hand side of (4), and fix $\varepsilon>0$. For each m.a.s.a. $\mathfrak{T}$ containing $\mathcal{Q}$, choose $T_{\mathscr{T}}$ in $\Re$ with $\left\|T-T_{\Re}\right\|<\delta+\varepsilon$. Let $\mathscr{K}$ denote the weak operator closed convex hull of $\left\{T_{\Re}\right\}$. If $\mathscr{K}$ were disjoint from $\mathcal{Q}$, then by the Hahn-Banach theorem, there is a weak operator continuous linear functional $\phi$ on $\mathscr{B}(\mathcal{K})$ which annihilates $\mathbb{Q}$ but is nonzero on all of $\mathcal{K}$. But by Lemma 3.3, there is a m.a.s.a. $\mathfrak{N}_{0}$ containing $\mathscr{Q}$ in the kernel of $\phi$. In particular, $\phi\left(T_{\mathscr{R}_{0}}\right)=0$ which is a contradiction. Hence $\mathscr{K}$ meets $\mathcal{Q}$. Let $A$ belong to the intersection $\mathscr{K} \cap \mathcal{Q}$. Then $\|T-A\| \leqslant \delta+\varepsilon$. Since $\varepsilon$ was arbitrary, $\operatorname{dist}(T, \mathbb{Q}) \leqslant \delta$. 
THEOREM 3.5. If $A$ is a von Neumann algebra such that $\mathbb{Q}$ or $\mathbb{Q}^{\prime}$ is abelian, then for all $T$ in $\mathscr{B}(\mathcal{H})$,

$$
\operatorname{dist}(T, \mathbb{Q}) \leqslant 2 \sup _{P \in \operatorname{lat} \mathbb{Q}}\left\|P^{\perp} T P\right\| \text {. }
$$

Proof. Lemma 3.1 suffices if $\mathcal{Q}^{\prime}$ is abelian. If $\mathscr{Q}$ is abelian and $\Re$ is a m.a.s.a. containing $\mathcal{Q}$, then lat $\mathfrak{N} \subseteq$ lat $\mathcal{Q}$, so by Lemma 3.4,

$$
\begin{aligned}
\operatorname{dist}(T, \mathscr{Q}) & =\sup _{\mathscr{N}} \operatorname{dist}(T, \mathscr{N}) \\
& \leqslant \sup _{\mathscr{N}} 2 \sup _{P \in \text { lat } \mathscr{T}}\left\|P^{\perp} T P\right\| \leqslant 2 \sup _{P \in \text { lat } Q}\left\|P^{\perp} T P\right\| .
\end{aligned}
$$

We conclude this section with a distance estimate for (possibly non-self-adjoint) algebras of normal operators.

THEOREM 3.6. If $\mathscr{B}$ is a unital weakly closed algebra of normal operators, then for all $T$ in $\mathscr{B}(\mathcal{H})$,

$$
\operatorname{dist}(T, \mathscr{B}) \leqslant 3 \sup _{P \in \text { lat } \mathscr{B}}\left\|P^{\perp} T P\right\| .
$$

Proof. By [7, Lemma 9.20], $\mathscr{B}$ is abelian. Let $\Re$ be a m.a.s.a. containing $\Re$. We may assume that $\|T\|=1$. Let $T_{0}$ be the operator produced in the proof of Lemma 3.1. If $U$ is a unitary in $\Re$, and $P$ belongs to lat $\mathscr{B}$, let $Q=U P U^{*}$. For $B$ in $\mathscr{B}$,

$$
Q^{\perp} B Q=U P^{\perp}\left(U^{*} B U\right) P U^{*}=U\left(P^{\perp} B P\right) U^{*}=0 .
$$

Thus $Q$ belongs to lat $\mathscr{B}$. Now

$$
\left\|P^{\perp}\left(U^{*} T U\right) P\right\|=\left\|\left(U P^{\perp} U^{*}\right) T\left(U P U^{*}\right)\right\|=\left\|Q^{\perp} T Q\right\| .
$$

Since $T_{0}$ belongs to the weakly closed convex hull of $\left\{U^{*} T U\right\}$,

$$
\sup _{P \in \text { lat } \mathscr{B}}\left\|P^{\perp} T_{0} P\right\| \leqslant \sup _{P \in \text { lat } \mathscr{B}}\left\|P^{\perp} T P\right\| .
$$

Also, by Lemma 3.1,

$$
\left\|T-T_{0}\right\| \leqslant 2 \sup _{P \in \text { lat } \mathscr{R}}\left\|P^{\perp} T P\right\| \leqslant 2 \sup _{P \in \text { lat } \mathscr{B}}\left\|P^{\perp} T P\right\| .
$$

We can complete the proof by proving that $\operatorname{dist}\left(T_{0}, \mathscr{B}\right) \leqslant \sup _{P \in \text { lat } \mathscr{B}}\left\|P^{\perp} T_{0} P\right\|$. Now $\operatorname{dist}\left(T_{0}, \mathscr{B}\right)=\sup \left|\phi\left(T_{0}\right)\right|$ where $\phi$ runs over all weak * continuous functionals on $\Re$ of norm one which vanish on $\mathscr{B}$. Let $\varepsilon>0$, and choose such a functional $\phi$ with $\operatorname{dist}\left(T_{0}, \mathscr{B}\right)<\left|\phi\left(T_{0}\right)\right|+\varepsilon$. Since $\mathscr{N}$ is maximal abelian, there are vectors $x$ and $y$ such that $\phi(M)=(M x, y)$ for all $M$ in $\Re$ and $\|x\|\|y\| \leqslant 1+\varepsilon$. Let $P_{0}$ be the orthogonal projection onto the closed span of $\mathscr{B} x$. Clearly, $P_{0}$ belongs to lat $\mathscr{B}$, $P_{0} x=x$, and $P_{0}^{\perp} y=y$. So

$$
\begin{aligned}
\operatorname{dist}\left(T_{0}, \mathscr{B}\right) & \leqslant\left|\left(T_{0} x, y\right)\right|+\varepsilon=\left|\left(P_{0}^{\perp} T_{0} P_{0} x, y\right)\right|+\varepsilon \\
& \leqslant\left\|P_{0}^{\perp} T_{0} P_{0}\right\|\|x\|\|y\|+\varepsilon \leqslant(1+\varepsilon) \sup _{P \in \text { lat } \mathscr{B}}\left\|P^{\perp} T_{0} P\right\|+\varepsilon .
\end{aligned}
$$

Thus, $\operatorname{dist}\left(T_{0}, \mathscr{B}\right) \leqslant \sup _{P \in \text { lat } \mathscr{B}}\left\|P^{\perp} T_{0} P\right\|$, and the theorem is proven.

4. Extensions of derivations. Let $\mathcal{Q}$ be an abelian von Neumann algebra. A derivation from $A$ to $\mathscr{B}(\mathcal{F})$ is a linear map $\Delta$ satisfying $\Delta(A B)=(\Delta A) B+A(\Delta B)$. It is well known that every bounded derivation from $Q$ into $\mathscr{B}(\mathcal{H})$ can be extended 
to an (inner) derivation of $\mathscr{B}(\mathscr{H})$. Here we give a slight strengthening of this using a technique developed in [1] and [3].

THEOREM 4.1. Let $\mathcal{P}$ be the set of projections in an abelian von Neumann algebra $\mathbb{Q}$. Suppose $\Delta$ is a map of $\mathscr{P}$ into $\Re(\mathcal{H})$ satisfying

(i) $\Delta(P+Q)=\Delta P+\Delta Q$ when $P Q=0$,

(ii) $\Delta(P Q)=(\Delta P) Q+P(\Delta Q)$ and

(iii) $\|\Delta P\| \leqslant M$ for all $P, Q$ in $\mathscr{P}$.

Then there is a $T$ in $\mathscr{B}(\mathcal{H})$ with $\|T\| \leqslant 2 M$ such that $\Delta=\Delta_{T} \mid \mathcal{P}$.

Proof. Let $\mathcal{L}=\left\{P_{j}, 1 \leqslant j \leqslant n\right\}$ be a finite subset of $\mathscr{P}$ with $\sum_{j=1}^{n} P_{j}=I$. Define $T_{\mathfrak{L}}=\Sigma_{i \neq j} P_{j} \Delta P_{i}$. The standard argument shows that $\Delta I=0$ and thus $\sum_{i=1}^{n} \Delta P_{i}=0$. Compute

$$
\begin{aligned}
T_{\mathfrak{L}} P_{m}-P_{m} T_{\mathfrak{L}} & =\sum_{i \neq j} P_{j}\left(\Delta P_{i}\right) P_{m}-P_{m} \sum_{i \neq j} P_{j} \Delta P_{i} \\
& =-\sum_{i \neq j}\left(\Delta P_{j}\right) P_{i} P_{m}-P_{m} \sum_{i \neq m} \Delta P_{i} \\
& =-\left(\sum_{j \neq m} \Delta P_{j}\right) P_{m}-P_{m}\left(\sum_{i \neq m} \Delta P_{i}\right) \\
& =\left(\Delta P_{m}\right) P_{m}+P_{m}\left(\Delta P_{m}\right)=\Delta P_{m} .
\end{aligned}
$$

Thus, for every projection $P$ in $\mathcal{L}^{\prime \prime}, T_{\mathfrak{L}} P-P T_{\mathfrak{L}}=\Delta P$. By Lemma 3.1,

$$
\operatorname{dist}\left(T_{\mathfrak{L}}, \mathcal{L}^{\prime}\right) \leqslant 2 \sup _{P \in \mathcal{P}^{\prime \prime}}\left\|T_{\mathfrak{L}} P-P T_{\mathcal{L}}\right\| \leqslant 2 \sup _{P \in \mathcal{L}^{\prime \prime}}\|\Delta P\| \leqslant 2 M .
$$

Choose an $A_{\mathfrak{L}}$ in $\mathcal{L}^{\prime}$ with $\left\|T_{\mathfrak{L}}-A_{\mathfrak{L}}\right\| \leqslant 2 M+1 / n$. Set $S_{\mathfrak{L}}=T_{\mathfrak{L}}-A_{\mathfrak{L}}$. Then $S_{\mathfrak{L}} P-$ $P S_{\mathfrak{L}}=\Delta P$ for every $P$ in $\mathcal{L}^{\prime \prime}$.

Since all finite subsets of $\mathscr{P}$ with sum $I$ form a directed set and the ball of radius $2 M+1$ is weakly compact, the net $\left\{S_{\mathcal{L}}\right\}$ has a limit point $T$. Clearly, $\|T\| \leqslant 2 M$ and $T P-P T=\Delta P$ for all $P$ in $P$.

It has come to our attention that Theorem 2.1 and Lemma 3.1 have been proven independently by Gilfeather and Larson [4], using similar methods.

ADDED IN PROOF. The author is greatly indebted to the referee for many improvements made on the manuscript.

\section{REFERENCES}

1. W. B. Arveson, Interpolation problems in nest algebras, J. Funct. Anal. 20 (1975), 208-233.

2. E. Christensen, Perturbations of operator algebras. II, Indiana Univ. Math. J. 26 (1977), 891-904.

3. K. R. Davidson, Commutative subspace lattices, Indiana Univ. Math. J. 27 (1978), 479-490.

4. F. Gilfeather and D. R. Larson, Nest subalgebras of von Neumann algebras: Commutants modulo compacts and distance estimates, preprint.

5. B. E. Johnson, Characterization and norms of derivations on von Neumann algebras, Lecture Notes in Math., vol. 725, Springer, Berlin and New York, 1979, pp. 228-236.

6. B. E. Johnson and S. K. Parrott, Operators commuting with a von Neumann algebra modulo the set of compact operators, J. Funct. Anal. 11 (1972), 39-61.

7. H. Radjavi and P. Rosenthal, Invariant subspaces, Springer-Verlag, Berlin, 1973.

8. J. T. Schwartz, $W^{*}$-algebras, Gordon and Breach, New York, 1967.

Bol'Shaya SERPUHOVSKaya UL. 31, KorPus 6, KV. 229A, Moscow 113093, U.S.S.R. 